\title{
TAGUNG
}

\section{Perspektiven für Europas Wirtschaftswachstum}

\author{
Marcus Kappler*
}

Die EU ist auf dem Weg, die in Lissabon definierten Zielsetzungen bei weitem zu verfehlen. Am deutlichsten wird dies im Hinblick auf das Ziel Wirtschaftswachstum. Dieser drohende Misserfolg ist besonders heikel im Hinblick auf die längerfristige Perspektive, in welcher der demographische Wandel in Europa aller Voraussicht nach ohnehin zu einer weiteren Dämpfung des Wirtschaftswachstums beitragen wird. Vor diesem Hintergrund befasste sich die Konferenz, zu der das Zentrum für Europäische Wirtschaftsforschung und der Arbeitskreis Europäische Integration eingeladen hatten, mit folgenden Fragen: Wie ist vergangenheitsbezogen die europäische Wachstumsperformance zu erklären? Welche Perspektive bietet sich im Hinblick auf die nächsten Jahrzehnte für das europäische Potenzialwachstum? Wie wirken sich unterschiedliche Wachstumsszenarien auf die Stabilität der öffentlichen Finanzen und der Sozialversicherungssysteme aus? Welche Instrumente stehen der Politik zur Verfügung, um das europäische Wachstumspotenzial positiv zu beeinflussen? Neben der Präsentation empirischer und modelltheoretischer Verfahren ging es auch darum, Handlungsempfehlungen für die Politik zu diskutieren.

\section{Der internationale Vergleich}

Den Auftakt der Konferenz übernahm Antonio Ciccone. Seinen Untersuchungen zufolge haben Länder mit einem hohen Humankapital in den 80er Jahren im Vergleich zu Ländern mit niedrigem Humankapital ein höheres Wachstum aufzeigen können. Die wirtschaftswissenschaftliche Literatur zeige einen signifikant positiven Effekt des Humankapitals auf die gesamtwirtschaftliche Produktion und auf

\section{Economic Growth in Europe}

Gemeinsame Tagung des Arbeitskreises Europäische Integration (AEI) und des Zentrums für Europäische Wirtschaftsforschung Mannheim GmbH (ZEW)

Mannheim, 23. und 24. Februar 2006

\section{Wissenschaftliche Leitung:}

Dr. Friedrich HEINEMANN, ZEW

Marcus KAPPLER, ZEW

\section{Welcome and Introduction}

Dr. Friedrich HEINEMANN, ZEW

Growth performance in the European Union

Human capital, the structure of production, and growth

Prof. Antonio CICCONE, Universitat Pompeu Fabra, Barcelona

The role of creative destruction for growth and job creation in Europe and the United States: a micro perspective

Stefano SCARPETTA, PhD, World Bank, Washington DC

The 'Lisbon Goal' of the EU: rhetoric or substance?

Prof. Dr. Wilhelm KOHLER, University of Tübingen

Economic growth in Europe: pursuing the Lisbon strategy

Fabienne ILZKOVITZ, European Commission, Brüssel

\footnotetext{
* Marcus Kappler, wiss. Mitarbeiter, Zentrum für Europäische Wirtschaftsforschung, Mannheim.
} 
die Beschäftigung auf. Ciccone untersuchte 40 Länder mit 37 Industrien ökonometrisch. Als Ergebnis seiner Untersuchungen kann festgehalten werden, dass in den Ländern, die einen hohen Bildungsstand der Bevölkerung vorweisen oder diesen Bildungsstand verbessern, höhere Wachstumsraten nachgewiesen werden konnten.

Ein hoher Bestand an Humankapital führe zu einer schnelleren technischen Adoption und somit zu einem schnelleren Einkommenswachstum. Damit könnten sich offene Volkswirtschaften mit einer höheren Humankapitalakkumulation schneller an humankapitalintensiveren Sektoren anpassen, was wiederum wachstumsfördernde Effekte auslöst. Auch eine längere Schulbildung kann bei funktionierenden Finanzmärkten und Schutz der Eigentumsrechte zu höheren Wachstumsraten führen. Aus diesem Grund sollten die einzelnen Länder darauf achten, ihre Investitionen in Bildung nicht zu unterschätzen, um diesen für Wachstum zentralen Faktor nicht zu vernachlässigen. Zwischen den OECD Ländern und den USA besteht eine Kluft im pro Kopf Einkommen, die sich seit den letzten Jahrzehnten vergrößert hat. Dies habe verschiedene, von Land zu Land differierende Ursachen. Produktivität, Arbeitseinsatz sowie Investitionen in Informations- und Telekommunikationstechnologien (ICT) bildeten dabei die Schlüsselfaktoren.

Stefano Scarpetta analysierte den Zusammenhang zwischen Regulierungsvorschriften und Wachstumsraten von OECD-Ländern. Hierbei stellte Scarpetta fest, dass niedrigere Markteintrittsbarrieren im Industriesektor zu höheren Wachstumsraten führen. Ein wesentlicher Grund sei, dass durch Markteintrittsbarrieren die Unternehmen im Industriesektor ihre Produktionsverfahren schneller an die internationale Spitze angleichen können. Weiterführende Reformen in anderen Sektoren hätten auch Produktivitätssteigerungen in der Herstellungsindustrie zur Folge. So profitiere die Herstellungsindustrie beispielsweise von Produktivitätsverbesserungen bei Vor- oder Zwischenprodukten.

\section{Europe's social model, aging and growth}

Growth and longevity from the industrial revolution to the future of an ageing society Prof. David de la CROIX, Université catholique de Louvain

Europe's social model - A reason of low growth?

Prof. Dr. Dr. h.c. Horst SIEBERT, Kiel Institute for World Economics and Johns Hopkins University, Bologna

\section{Fiscal policy and growth}

Public debt, public investment and economic growth

Prof. Dr. Alfred GREINER, University of Bielefeld

Public sector efficiency: evidence for the new EU member states and emerging markets

António AFONSO, PhD, European Central Bank and ISEG/UTL-Technical University of Lisbon

New empirical and theoretical approaches

On the relation between government size and economic development: some methodological and econometric remarks

Prof. Dr. Gebhard KIRCHGÄSSNER, University of St. Gallen

Multi-country endogenous growth models Prof. Dr. Lutz ARNOLD, University of Regensburg

A meta-analysis of beta-convergence: the legendary two-percent

Maria ABREU, Cambridge-MIT Institute, University of Cambridge

Policy and regional growth: drivers of regional productivity development

Martin EICHLER and Michael GRASS, BAK Basel Economics

Once again: investigating hours worked Marcus KAPPLER, ZEW 
Untersuchungen auf Firmenebene seien in der Lage, Erkenntnisse der gesamtwirtschaftlichen Analyse gut widerzuspiegeln. Je länger die Unternehmen der Industriestaaten auf dem Markt sind, umso schneller wachsen sie im Zeitverlauf. Die Erklärung liege darin, dass bei geringen Eintrittsbarrieren die Arbeitskräfte anfangs weniger produktiv seien, aber ihre Produktivität über die Jahre erhöhten. Die Unternehmen expandieren, wobei eine höhere Produktivität dann zu mehr Wachstum führe. Arbeitet ein Unternehmen nicht effizient werde es vom Markt aussortiert, was von niedrigen Austrittsbarrieren unterstützt wird. Produktmarktregulierungen verhinderten Aufholprozesse, während geringere Eintrittskosten mehr Unternehmen in den Wettbewerb ziehen, die versuchten, sich gegenseitig in der Produktivität zu überbieten.

Anhand von Handels- und Wachstumstheorien untersuchte Wilhelm Kohler mögliche „Fallgruben" und Kurzzeiteffekte der Wirtschaftspolitik im Hinblick auf das Erreichen des Lissabon-Ziels. Er wies darauf hin, dass die Politiken zwar rechtskräftig seien, jedoch das Paradigma des internationalen Wettbewerbs oft falsch verstanden werde. Die Wohlfahrt werde zum Beispiel an der Produktivität der Beschäftigten gemessen. Betrachte man jedoch die Produktivität allein könne man keine genaue Aussage über die Wohlfahrt machen.

Das Wachstumsmodell des US-Ökonomen Lucas zeigt, wie die Politik Fördergelder und Steuern in Richtung lernintensiver Industrien dirigieren und dafür sorgen kann, dass die ,Gewinner' auch weiterhin gut auf dem Markt agieren können. Die Determinanten Arbeitslosigkeit und pro Kopf Einkommen erklären in diesem Modell maßgeblich das Wachstum. Die EU könne helfen, dass der Wohlfahrtsstaat reformiert, die Arbeitsmarktrigiditäten verringert, die öffentlichen Finanzen im Auge behalten und das Bildungssystem optimiert werde. Zur Verwirklichung sollte die Kommunikation und Koordination zwischen den Mitgliedsländern verstärkt werden.

Fabienne Ilzkovitz, beschäftigte sich mit dem ,Fortsetzen der Lissabon Strategie‘. Die alte
Strategie war darauf ausgelegt, die Lücke zwischen den USA in den Bereichen BIP pro Kopf, Beschäftigungsrate, Arbeitsstunden und stündliche Arbeitsproduktivität pro Beschäftigten zu schließen. Dies wurde bisher nicht erreicht. Gerade in Zeiten des demographischen Wandels und der Globalisierung sei es nötig, wettbewerbsfähig zu bleiben. Aus diesem Grunde wurden wichtige Reformen im Hinblick auf die Lissabon-Strategie zur Steigerung des BIP, zur Aufrechterhaltung des Sozialmodells und der Erhaltung der Umwelt eingeleitet. Hierzu zählten Arbeitsmarkt-, Produktmarkt-, Investitions- und Kapitalmarktreformen. Positiv bisher zu erwähnen sei, dass Strukturreformen, bessere Überwachungsinstrumente und eine Wettbewerbsagenda geschaffen sowie erste Schritte zu einer koordinierten Politik veranlasst wurden. Negative Aspekte seien bisher die zu hohe Anzahl der Prioritäten, die zeitliche Verzögerungen in mehreren Bereichen, die zu komplexe Koordination und die nicht ausreichende Kommunikation gewesen.

Die Neue Lissabon Strategie ziele mehr auf Wachstum und Beschäftigung ab. Zudem solle die Wissensversorgung verbessert werden sowie die Innovationstätigkeit angeregt werden, wodurch Europa wieder attraktiver für Investoren werden könne. Damit jedoch Fortschritt erzielt werden könne, seien nicht nur die EU, sondern auch die einzelnen Länder mit Hilfe ihrer nationalen Reformprogramme gefragt. Dabei spielten vor allem die öffentlichen Finanzen, Arbeitsmarktpolitik, Forschung und Entwicklung, Umweltschutz sowie Produktmarktregulierungen eine wesentliche Rolle. Entscheidend sei es, die Mitgliedstaaten von der Notwendigkeit von Reformen zu überzeugen, da die Langzeiteffekte, auch auf nationaler Ebene, positiven Einfluss auf Wachstum, soziale Absicherung und Umweltschutz haben werden.

Demographische Erklärungsansätze und kontinentaleuropäische Sozialmodelle

David de la Croix führte in seinem Beitrag Produktivität und Wachstum auf Bevölke- 
rungswachstum, eine große Anzahl von Kindern und schließlich auf die Bevölkerungsgröße zurück. Um diesen Zusammenhang herzuleiten, präsentierte er ein demographisch-ökonomisches Modell, in dem er sich zunächst an die endogenen Wachstumstheorien anlehnte, nach welchen eine ansteigende Lebenserwartung zu einer längeren Schulbildung und folglich zu höherem Humankapital in einer Volkswirtschaft führe, was wiederum positive Auswirkungen auf das Wachstum habe. Anhand eines Modells demographischer Dividenden könne das Wirtschaftswachstum mit Hilfe der Größe und Struktur der Bevölkerung erklärt werden. Mit zunehmendem Alter nimmt die Produktivität der Bevölkerung zu, während ab einem Alter von 65 Jahren die älteren Beschäftigten weniger produktiv als jüngere werden. Kombiniere man nun beide Modelle miteinander und nutzt die jeweiligen Stärken, könnten genauere Prognosen für das zukünftige Wachstum einer Volkswirtschaft erstellt werden, so de la Croix. Über den demographischen Wandel und den Ausbildungsstand lasse sich folglich das Langzeitwachstum des pro Kopf Einkommens erklären.

Besteht eine Verbindung zwischen Wachstum und sozialer Absicherung? Horst Siebert befasste sich mit dieser zentralen Frage. Siebert ging davon aus, dass ein Zielkonflikt zwischen diesen beiden Problemen bestünde. Für den Arbeitsmarkt zeigte er, durch welche institutionellen Bestimmungen das Wachstum gehemmt und die Arbeitsnachfrage von Unternehmen reduziert wird. Hierzu zählten der Arbeitschutz, Arbeitslosenhilfe (Arbeitslosengeld), Lohnnebenkosten, Mindestlöhne und Tarifverträge. Folglich gingen die Einnahmen bei geringerer Beschäftigung für den Staat zurück, während sich gleichzeitig die Kosten der sozialen Absicherung erhöhten. Ein weiterer innovativer Ansatz wäre es, Humankapital dem freien Wettbewerb auszusetzen, das heißt die Universitäten untereinander konkurrieren $\mathrm{zu}$ lassen sowie Unternehmen finanzielle Belohnungen für Innovationen zukommen $\mathrm{zu}$ lassen.
Leider sei die Budgetpolitik der „Großen Drei“, Frankreich, Deutschland und Italien, nicht mehr unabhängig von ihrer Sozialpolitik. Davon sei die Nachfrage und auch die Kapitalbildung betroffen, die einen wichtigen Bestandteile des Wachstum darstellten, was auch eine Gefahr für die Stabilität der Gemeinschaftswährung darstellen könnte. Fördergelder verzerrten das Marktgleichgewicht und störten damit marktdynamische Entwicklungen. Das Mitspracherecht der Arbeitnehmer in großen Unternehmen sowie beispielsweise Dreijahresverträge für Manager würde dafür sorgen, dass wichtige Entscheidungen von persönlichen Interessen geprägt werden. Aus diesem Grund gelangte Siebert zu dem Fazit, dass weniger politischer Eingriff auf Märkte sowie eine grundlegende Reform der sozialen Sicherungssysteme notwendig seien. Vor allem „Old Europe“ stünde hier vor einer schweren Aufgabe.

\section{Die Bedeutung des Staates}

Dem Staat stehen Steuereinnahmen und Kreditverschuldung zur Verfügung, um Staatsausgaben wie Investitionen und Zinszahlungen zu tätigen. Nach Alfred Greiners Modell führt eine geringere Verschuldung zu mehr Produktion und $\mathrm{zu}$ einer größeren Wachstumsrate. Öffentliche Investitionen verstärkten zudem die Wachstumseffekte. Steige die Neuverschuldung stark an, komme es durch mehr Investitionstätigkeit auch zu höheren Wachstumsraten, die im Vergleich zur Neuverschuldungsrate allerdings geringer ausfalle. Durch erhöhte Zinszahlungen komme ein negativer Effekt zum Tragen. Je größer dabei das betrachtete Zeitfenster sei, umso geringer steige die Neuverschuldung, aber auch der Konsum. Insgesamt könne festgehalten werden, dass höhere Schulden über die gesamte Zeit zu weniger Wachstum führten.

Mit der Effizienz im öffentlichen Sektor beschäftigt sich António Afonso in seiner Studie, die er auf der Konferenz vorstellte. Darin werden Fragen behandelt, ob ein Vorgang als effizient betrachtet werden könne, wenn ein 
vorgegebenes Ziel punktgenau erreicht wird, obwohl dies viele Ressourcen kostet oder man ein Ziel nur annäherungsweise erreicht, dafür jedoch mit weniger Aufwand. Mit den Instrumenten des „Public Sector Performance Index“ (PSP) und der „Data Envelopment Analyses" (DEA) gab Afonso Antworten auf diese Problemstellung. Der PSP gibt dabei an, welche Länder leistungsstark agieren, während mit der DEA die Ineffizienz zwischen Ressourceneinsatz und Output aufgezeigt wird. Dies geschehe, indem eine theoretisch mögliche Produktionsgrenze angenommen wird, wodurch es für einige Länder möglich wäre, ihren bisherigen Output mit bis zu 45 Prozent weniger Ressourceneinsatz zu erreichen. Auf der anderen Seite würden meist nur circa 75 Prozent des theoretisch möglichen Outputs erreicht. Einen signifikanten Einfluss auf die Effizienz des öffentlichen Sektors übten vor allem die Faktoren ,Wahrung von Eigentumsrechten“, „BIP pro Kopf“, „Ausbildung der Bevölkerung“ sowie „Regierungsfähigkeit“ aus. Hier könnten in vielen Ländern richtungweisende Entscheidungen getroffen werden, damit ein effizienter Umgang mit öffentlichen Ressourcen gewährleistet wird.

\section{Methodische Vorgehensweisen zur Erfassung von Zusammenhängen}

Gebhard Kirchgässner analysierte den $\mathrm{Zu}$ sammenhang zwischen der Größe des öffentlichen Sektors und der wirtschaftlichen Entwicklung eines Landes. Er ging damit der Frage auf den Grund, ob intensive Eingriffe der jeweiligen Regierung in das Wirtschaftsgeschehen verantwortlich für die unterdurchschnittliche Wachstumsentwicklung in einigen europäischen Ländern seien. Eine wichtige Voraussetzung für solche Analysen sei vor allem, eine vergleichbare Größe für die Aktivität einer Regierung definieren zu können, da nur so Unterschiede einzelner Länder, wie zum Beispiel im Gesundheitsoder Rentensektor, berücksichtigt werden könnten. Bereits hier zeigten sich schon erhebliche methodische Schwierigkeiten. Ent- gegen der Meinung vieler Ökonomen, wonach eine Reduktion der staatlichen Aktivität zu höherem Wachstum führe, zeigte Kirchgässner auf, dass es auch empirisch nachgewiesene positive Zusammenhänge zwischen staatlicher Aktivität und Wachstum gebe. Genauso existierten auch Länder, die trotz niedriger Staatsaktivität ein vergleichsweise bescheidenes Wachstum aufweisen würden. Als Beispiel für eine hohe Staatsaktivität und zeitgleich hohes Wirtschaftswachstum nannte Kirchgässner die skandinavischen Länder (,Scandinavian Miracles“). Den Grund hierfür sah Kirchgässner in der effizienten Bereitstellung der von der Regierung angebotenen Dienstleistungen. Zugleich nannte er das ,Suisse Puzzle" und damit die Tatsache, dass die Schweiz trotz niedriger Staatsaktivität seit 15 Jahren nur ein unterdurchschnittliches Wirtschaftswachstum aufweist.

Die Betrachtung zahlreicher empirischer Studien lasse kein einheitliches Bild erkennen, wie sich das Eingreifen einer Regierung in das Wirtschaftsgeschehen auf das Wachstum auswirke. Es wurden sowohl signifikant positive als auch signifikant negative Zusammenhänge nachgewiesen. Gründe hierfür sah Kirchgässner unter anderem im nichtlinearen Zusammenhang zwischen den beiden Variablen der Abhängigkeit von den sozialen Sicherungssystemen in einer Volkswirtschaft und des Einflusses der Ausgaben des Staates beziehungsweise des Steuersystems. Sowohl in einer Analyse verschiedener Länder als auch in einer Untersuchung verschiedener Schweizer Kantone kam Kirchgässner nicht zu dem Schluss, dass eine Reduktion der staatlichen Aktivität das Wachstum in einer Volkswirtschaft positiv beeinflusst. Politische Empfehlungen zur Reduktion der Einflussnahme der Regierung könnten somit laut Kirchgässner nicht auf empirischen Analysen beruhen.

Der Frage nach dem Effekt ökonomischer Integration in endogenen Wachstumsmodellen ist Lutz Arnold nachgegangen. Arnold analysierte endogene Wachstumsmodelle offener Volkswirtschaften mit besonderem Augen- 
merk auf Übergangsdynamiken. Dieser Sachverhalt wurde laut Arnold bisher nur in wenigen endogenen Wachstumsmodellen berücksichtigt. Im Laufe seines Vortrages ging Arnold kurz auf die bereits bekannten endogenen Wachstumsmodelle unter anderem von namhaften Ökonomen wie Solow, Lucas und Jones ein. Weiter erklärte Arnold, wie sein Modell sowohl auf den neoklassischen Wachstumsmodellen als auch auf den neueren endogenen Wachstumsmodellen aufbaut. Als besonders wichtige Variable in der Wachstumsempirie hob er das Humankapital sowie Forschung und Entwicklung (FuE) hervor, für letztere seien Investitionen in FuE zur Produktinnovation von Investitionen zur Qualitätsverbesserung zu unterscheiden.

Zunächst skizzierte Arnold ein stark fokussiertes, hypothetisches Modell, in dem es keine nationalen Grenzen gebe. Die ganze Welt bestehe somit aus einer zusammenhängenden Volkswirtschaft. Das Rückgrat dieses Wachstumsmodells stellten die vier zu Grunde liegenden Produktionsfunktionen (für Endprodukt, Zwischenprodukt, FuE sowie Humankapital) dar. Den Durchbruch in seiner Analyse sah Arnold in der Erkenntnis, dass es möglich sei, Übergangsdynamiken in einer offenen Volkswirtschaft aufbauend auf den Annahmen der Modelle für geschlossene Volkswirtschaften zu untersuchen. Dies sei dadurch möglich, dass man in die integrierte Modellwelt die nationalen Grenzen einführt und nachweist, dass die für die hypothetische Modellwelt getroffenen Annahmen weiterhin ihre Gültigkeit besitzen. In der anschließenden Diskussion hob Arnold noch einmal hervor, dass es sich um eine sehr modelltheoretische Analyse handelt. Es sei nicht der Anspruch, konkrete Empfehlungen zum Beispiel für die Politik zu geben. Vielmehr sei der Vorteil dieser Vorgehensweise, „eine Theorie im Hinterkopf für praktische Analysen zu haben“.

In der Wachstumsökonomik spielt der Begriff der Konvergenz eine zentrale Rolle. Er gibt den theoretischen und häufig beobachteten
Sachverhalt wieder, dass ärmere Länder zu den Einkommensniveaus der reicheren Länder aufschließen. Maria Abreu hat zusammen mit ihren Koautoren mittels einer so genannten ,Meta-Analyse' die Frage untersucht, ob ein Zusammenhang zwischen Wachstum und ursprünglichem Einkommen von Volkswirtschaften bestehe. Der Zusammenhang dieser Größen sei in der Wachstumstheorie auch als „Beta-Konvergenz“ geläufig. Die Meta-Analyse, die oft in der Medizin oder auch in den Verhaltenswissenschaften angewandt werde, finde auch in den Wirtschaftswissenschaften zunehmend Verbreitung. Dabei würden durch frühere Studien gewonnene Ergebnisse über ein und dieselbe Variable im Hinblick auf Konsenserkenntnisse untersucht. In ihrer konkreten Untersuchung ging es den Autoren auch darum, voneinander abweichende Ergebnisse zur Konvergenzgeschwindigkeit bisheriger empirischer Studien zu erklären.

Abreu hat im Rahmen der Meta-Analyse die aus zahlreichen Studien hervorgegangene Beta-Konvergenz in Höhe von zwei Prozent als ,natürliche Konstante“ untersucht. Hierfür seien per Zufallsprinzip ausgewählte Studien aus der umfangreichen Konvergenzliteratur analysiert worden. Die Stichprobe resultierte in insgesamt 610 geschätzten Konvergenzraten aus 48 empirischen Studien. In ihrer Analyse seien dann aus den so ermittelten Daten ein so genanntes ,Meta-Regressionsmodell ${ }^{\circ}$ entwickelt worden, welches als erklärende Variablen Dateneigenschaften der Stichprobenstudien, Merkmale der verwendeten Schätzmethoden sowie weitere konditionierende Variablen aus früheren Studien einbezog. Mit einem aus diesem Regressionsmodell resultierendem Schätzwert für die BetaKonvergenz in Höhe von 2,4 Prozent liege man ziemlich nahe am ,erwarteten“ Wert von zwei Prozent. $\mathrm{Zu}$ beachten sei hierbei allerdings die Auswirkungen des so genannten ,Publication Bias' auf die der Studie zugrunde liegenden Veröffentlichungsdaten. Hierbei handele es sich um das Phänomen, dass Ergebnisse für die Beta-Konvergenz, die statis- 
tisch nicht signifikant von Null verschieden sind, gar nicht erst veröffentlicht werden.

Martin Eichler und Michael Grass haben sich im Rahmen ihres Forschungsprojektes mit den Wachstumsraten und Wachstumsmotoren in unterschiedlichen europäischen Regionen befasst. Eichler wies darauf hin, dass Regionen in einer Volkswirtschaft durchaus sehr heterogene Strukturen und Wachstumsentwicklungen aufwiesen. Somit würde die Betrachtung auf regionaler Ebene auch in der Politikberatung an Bedeutung gewinnen. Eichler und Grass haben in einem Datensatz bestehend aus 120 europäischen Regionen für den Zeitraum von 1980 bis 2003 als Politikvariablen Innovationskapazitäten, Steuerniveaus, infrastrukturelle Erreichbarkeit und Regulierungen identifiziert. Weitere lokale Faktoren wie die Branchenstruktur, geographische Lage und historisches Wachstum wurden ebenfalls als erklärende Variablen für das unterschiedliche Wachstum verschiedener Regionen herangezogen.

Als wesentliche Ergebnisse einer ökonometrischen Analyse sei festzuhalten, dass die oben genannten Variablen alle signifikant zum Wachstum in den verschiedenen Regionen beitragen würden. Regionen könnten durch einen sensiblen und angepassten Politik-Mix einen direkten Einfluss auf das Wachstum nehmen. Besonders die Besteuerung von hoch qualifizierten Arbeitnehmern wirke sich negativ auf das Wachstum in einer Region aus. Als Handlungsempfehlung ließe sich das Anwerben und Binden von eben diesen Personen geben. Dies sei neben der Kontrolle der steuerlichen Belastung auch über andere Faktoren, wie zum Beispiel der Lebensqualität in einer Region, möglich. Eichler betonte darüber hinaus, dass Politik Zeit brauche, viele politische Maßnahmen hingegen nur verzögert Erfolge aufwiesen. In diesem Zusammenhang sei es wichtig, dass zukünftige Erfolge ausführlich potenziellen Investoren sowie der Bevölkerung dargestellt und kommuniziert würden.
Die geleisteten Arbeitsstunden in einer Volkswirtschaft werden im Rahmen empirischer Studien in der Wachstumsökonomik sehr häufig als erklärende Variable herangezogen. So bauen beispielsweise die Schätzungen von Arbeitsangebotselastizitäten oft auf den geleisteten Arbeitsstunden auf. Marcus Kappler ging im Rahmen seines Vortrages auf die Eigenschaften der Zeitreihe der geleisteten Arbeitsstunden insbesondere der Nicht-Stationarität der Zeitreihe ein. Im Gegensatz zu stationären Zeitreihen zeigen nicht-stationäre Daten keine automatische Rückkehr zu einem langfristig stabilen Mittelwert im Zeitverlauf. Kappler zeigte, dass sich mit Hilfe verschiedener Testmethoden für OECD-Länder die Hypothese absichern lasse, dass die Zeitreihe für geleistete Arbeitsstunden nicht stationär verlaufen würde. Die Eigenschaften einer nicht-stationären Zeitreihe unterscheiden sich stark von der einer stationären Zeitreihe und im Rahmen einer empirischen Analyse könne die falsche Annahme von Stationarität zu erheblichen Verzerrungen in den Ergebnissen führen. Kappler testete sowohl für die Länder isoliert als auch im Rahmen einer Panel Daten Analyse die Zeitreihen auf Nicht-Stationarität.

\section{Ausblick}

Die Vorträge stellten in unterschiedlichen Ansätzen und methodischen Vorgehensweisen Einsichten in die Wachstumsentwicklungen in Deutschland und den Industrieländern dar, welche insbesondere im Hinblick auf die Neuformulierung der Lissabon-Strategie noch an Bedeutung für die wirtschaftspolitische Orientierung gewonnen haben. Auf der Konferenz sorgten die unterschiedlichen Denkansätze der Teilnehmer und Referenten für einen fruchtbaren Austausch von Erfahrungen und Erkenntnissen, von dem alle Anwesenden für ihre weiteren praxisorientierten oder forschungsfokussierten Arbeiten profitieren konnten. 